\title{
Suppression of EGFR/STAT3 activity by lupeol contributes to the induction of the apoptosis of human non-small cell lung cancer cells
}

\author{
TAE-RIN MIN ${ }^{1 *}$, HYUN-JI PARK ${ }^{1 *}$, KI-TAE HA $^{2}$, GYOO-YONG CHI ${ }^{1}$, \\ YUNG-HYUN CHOI ${ }^{3}$ and SHIN-HYUNG PARK ${ }^{1}$ \\ ${ }^{1}$ Department of Pathology, College of Korean Medicine, Dong-Eui University, Busan 47227; \\ ${ }^{2}$ Department of Korean Medical Science, School of Korean Medicine and \\ Healthy Aging Korean Medicine Research Center, Busan National University, \\ Yangsan, Gyeongsangnam-do 50612; ${ }^{3}$ Department of Biochemistry, \\ College of Korean Medicine, Dong-Eui University, Busan 47227, Republic of Korea
}

Received January 22, 2019; Accepted May 7, 2019

DOI: $10.3892 /$ ijo.2019.4799

\begin{abstract}
The aim of this study was to investigate the underlying mechanisms responsible for the anticancer effects of lupeol on human non-small cell lung cancer (NSCLC). MTT assay and Trypan blue exclusion assay were used to evaluate the cell viability. DAPI staining and flow cytometric analysis were used to detect apoptosis. Molecular docking and western blot analysis were performed to determine the target of lupeol. We found that lupeol suppressed the proliferation and colony formation of NSCLC cells in a dose-dependent manner. In addition, lupeol increased chromatin condensation, poly(ADP-ribose) polymerase (PARP) cleavage, sub-G1 cell populations, and the proportion of Annexin V-positive cells, indicating that lupeol triggered the apoptosis of NSCLC cells. Notably, lupeol inhibited the phosphorylation of epithelial growth factor receptor (EGFR). A docking experiment revealed that lupeol directly bound to the tyrosine kinase domain of EGFR. We observed that the signal transducer and activator of transcription 3 (STAT3), a downstream molecule of EGFR, was also dephosphorylated by lupeol. Lupeol suppressed the nuclear translocation and transcriptional activity of STAT3 and downregulated the expression of STAT3 target genes. The constitutive activation of STAT3
\end{abstract}

Correspondence to: Professor Shin-Hyung Park, Department of Pathology, College of Korean Medicine, Dong-Eui University, 52-57 YangJeong-ro, Busanjin-gu, Busan 47227, Republic of Korea E-mail: omdpark@deu.ac.kr

*Contributed equally

Key words: lupeol, epithelial growth factor receptor, epithelial growth factor receptor tyrosine kinase inhibitor resistance, signal transducer and activator of transcription 3, apoptosis, non-small cell lung cancer by STAT3 Y705D overexpression suppressed lupeol-induced apoptosis, demonstrating that the inhibition of STAT3 activity contributed to the induction of apoptosis. The anticancer effects of lupeol were consistently observed in EGFR tyrosine kinase inhibitor (TKI)-resistant H1975 cells (EGFR L858R/T790M). Taken together, the findings of this study suggest that lupeol may be used, not only for EGFR TKI-naïve NSCLC, but also for advanced NSCLC with acquired resistance to EGFR TKIs.

\section{Introduction}

Lung cancer exhibits the highest mortality rate among all types of cancer worldwide. Even though chemotherapy is one of the standard therapies for lung cancer, $<20 \%$ of patients treated with chemotherapy live $>5$ years (1). This dismal number indicates that advances in lung cancer treatment remain inadequate compared to other types of cancer. The poor prognosis of patients with lung cancer is mainly derived from the low response rate and resistance to current chemotherapeutics (2). As non-small cell lung cancer (NSCLC) is the most common type of lung cancer (3), the development of more effective and advanced therapeutic strategies for the treatment of NSCLC is fundamental in order to improve the poor prognosis of patients with lung cancer.

Epidermal growth factor receptor (EGFR) is a transmembrane receptor tyrosine kinase protein which belongs to the ErbB family. Upon the binding of a ligand, such as epidermal growth factor (EGF), the intracellular domain of EGFR is phosphorylated and activates downstream signal transduction pathways, including RAS/RAF/MEK/mitogen-activated protein kinase (MAPK), phosphoinositide 3-kinase (PI3K)/Akt, and signal transducer and activator of transcription (STAT) signaling pathways. These signal transductions finally result in cell proliferation and in the inhibition of apoptosis (4). The overexpression of EGFR has been implicated in the pathogenesis of NSCLC $(5,6)$. Studies have reported that EGFR overexpression in NSCLC is associated with a reduced overall survival, chemoresistance and frequent lymph node metastasis (7-11). In addition, a quarter of NSCLCs 
cases possess activating mutations in the tyrosine kinase domain of EGFR (12). These mutations sensitize NSCLCs to EGFR receptor tyrosine kinase inhibitors (TKIs), such as gefitinib and erlotinib (13-15). However, patients ultimately develop acquired resistance against these drugs. The most common mechanism of resistance is a secondary T790M mutation in EGFR exon 20 (16,17). Thus, the identification of novel drugs to effectively suppress the activity of EGFR, not only in naïve NSCLCs, but also in EGFR TKI-resistant NSCLCs is imperative.

STAT3 is a transcription factor recognized as a key oncogenic factor driving tumor development and progression. STAT3 is activated by phosphorylation at tyrosine 705 or serine 727 via interleukin (IL)-6 receptor (IL-6R), growth factor receptors, and non-receptor tyrosine kinase, such as Src (18). The activation of STAT3 mediates a variety of cellular functions, including cell proliferation, differentiation, angiogenesis, metastasis, and drug resistance (19). Studies have reported that STAT3 was activated in NSCLC and a high phosphorylation level of STAT3 was a strong predictor of poor prognosis in NSCLC (19-21). Specifically, STAT3 signaling has been related to the development of resistance to EGFR TKIs (22-27). Therefore, aberrant STAT3 phosphorylation appears to be a potential therapeutic target for NSCLC.

Lupeol (chemical structure shown in Fig. 1A) is a dietary triterpenoid present in various types of fruits, vegetables and medicinal plants. Lupeol has been reported to exhibit strong antioxidant, anti-inflammatory, anti-microbial, anti-arthritic, anti-diabetic and anti-malarial activities (28). Moreover, lupeol has been shown to exert anticancer effects in various cancer cells. The suppression of tumorigenesis, the induction of apoptosis, cell cycle regulation, chemosensitization and the enhancement of the cytotoxic function of natural killer cells have been reported as the mechanisms of the anticancer effects of lupeol (7,29-37). Notably, lupeol has been shown to suppress EGFR activity in oral squamous cell carcinoma and gallbladder carcinoma $(36,37)$. It has also been shown to inhibit the STAT3 signaling cascade in hepatocellular carcinoma cells (7). In NSCLC, lupeol has been reported to downregulate COX2 and mTOR/PI3K/AKT pathways to induce apoptosis $(33,34)$. However, the regulatory effects of lupeol on the EGFR/STAT3 signaling pathway in human NSCLC cells have not yet been elucidated, at least to the best of our knowledge. Thus, in the current study, we investigated the mechanisms responsible for the anticancer activity of lupeol in human NSCLC cells, focusing on the regulation of EGFR/STAT3 activity. We also aimed to verify whether lupeol exerts anticancer effects on NSCLC cells that are resistant to EGFR TKIs.

\section{Materials and methods}

Cell lines and cell culture. The H1299, A549, H460, H292 human NSCLC cell lines and WI38 human lung fibroblast were purchased from the American Type Culture Collection (ATCC). The H1975 human NSCLC cell line was kindly supplied by professor Ho-Young Lee (College of Pharmacy, Seoul National University). The cells were grown in RPMI-1640 (WelGENE) supplemented with 10\% fetal bovine serum (FBS, WelGENE) and $1 \%$ antibiotics (WelGENE) at $37^{\circ} \mathrm{C}$ in a humidified incubator under $5 \% \mathrm{CO}_{2}$.
Reagents and antibodies. Lupeol was purchased from ChemFaces and was dissolved in dimethyl sulfoxide (DMSO; Sigma-Aldrich).TrypanbluewaspurchasedfromWelGENE, and MTT [3-(4,5-dimethylthiazol-2-yl)-2,5-diphenyltetrazolium bromide] from Duchefa. Hematoxylin, propidium iodide (PI), paraformaldehyde and 4,6-diamidino-2-phenylindole (DAPI) were obtained from Sigma-Aldrich. Primary antibodies against phospho-EGFR (Y1068, \#2234S), EGFR (\#4267S), phospho-STAT3 (Y705, \#9145S), STAT3 (\#9139S), phospho-AKT (S473, \#4060S), phospho-ERK (T202/Y204,\#9106S), cleaved caspase-3 (\#9661S) and cleaved poly(ADP-ribose) polymerase (PARP, \#5625S) were purchased from Cell Signaling Technology. The other primary antibodies including AKT (\#sc-5298), ERK (\#sc-514302), $\beta$-actin (\#sc-47778), $\alpha$-tubulin (\#sc-5286), Lamin B (\#sc-374015), survivin (\#sc-17779) and cyclin D1 (\#sc-450) were obtained from Santa Cruz Biotechnology. Goat anti-mouse secondary antibody was purchased from Bethyl Laboratories and goat anti-rabbit secondary antibody was purchased from Enzo Life Sciences.

Cell viability assay. For the MTT assay, $3 \times 10^{3}$ cells were seeded onto 96-well plates and treated with lupeol (10-100 $\mu \mathrm{M})$ for various time periods (24-72 h) or treated with erlotinib (LC Labs; $10-100 \mu \mathrm{M}$ ) for $72 \mathrm{~h}$. MTT solution was added to the media at a concentration of $0.4 \mathrm{mg} / \mathrm{ml}$ followed by incubation for $4 \mathrm{~h}$ at $37^{\circ} \mathrm{C}$. The media were then aspirated and $100 \mu \mathrm{l}$ of DMSO were added to each well to dissolve the formazan. The absorbance values at $540 \mathrm{~nm}$ were measured using a microplate reader (SpectraMax M3; Molecular Devices). For the trypan blue exclusion assay, $2 \times 10^{4}$ cells were seeded in 12 -well plates and treated with lupeol at 50 or $100 \mu \mathrm{M}$ for $72 \mathrm{~h}$. The cells were then collected and stained with $0.4 \%$ trypan blue solution at a final concentration of $0.1 \%$. The number of viable cells was evaluated by counting the unstained cells using a hemocytometer under a microscope (Leica).

Anchorage-dependent and-independent colony formation assay. For the anchorage-dependent 2D colony formation assay, $3 \times 10^{2}$ cells were seeded in 12 -well plates and treated with lupeol for 2 weeks. The medium was changed every 3 days. The colonies were fixed with $100 \%$ methanol for $5 \mathrm{~min}$ and stained with hematoxylin for $30 \mathrm{~min}$ at room temperature. Images of the stained colonies were acquired using a digital camera (Canon) and the number of colonies was counted using ImageJ software. For the anchorage-independent colony formation assay (soft agar assay), 4\% SeaPlaque agarose (Lonza) dissolved in PBS was melted and mixed with warm media to yield $1 \%$ bottom agar. Bottom agar $(1 \mathrm{ml})$ was then added to 24 -well plates and allowed to solidify at room temperature. The cells $\left(1 \times 10^{3}\right)$ were suspended in $0.5 \mathrm{ml}$ of top agar $(0.4 \%)$ and plated onto the bottom agar. The plate was kept at room temperature until the top agar solidified. The cells were then treated with lupeol at 25, 50 and $100 \mu \mathrm{M}$ for 2 weeks and the medium was changed every 3 days. The colonies were stained with MTT solution (final concentration, $0.5 \mathrm{mg} / \mathrm{ml}$ ) for $2 \mathrm{~h}$ at $37^{\circ} \mathrm{C}$. Images of the stained colonies were acquired using a digital camera (Canon) and the number of colonies was counted using ImageJ software. 
DAPI staining. The cells $\left(1 \times 10^{5}\right)$ were seeded in 6-well plates and treated with lupeol at $100 \mu \mathrm{M}$ for $72 \mathrm{~h}$. The cells were then harvested, fixed with $3.7 \%$ paraformaldehyde, and attached to slide glasses using a cytospin (Shandon). After staining with DAPI solution $(2.5 \mu \mathrm{g} / \mathrm{ml})$ for $20 \mathrm{~min}$ at room temperature in the dark, the attached cells were washed with PBS and distilled water and mounted with aqueous mounting medium (Crystal Mount). The morphology of the nuclei was observed under a fluorescence microscope (Carl Zeiss) at x200 magnification.

Flow cytometric analysis. The cells $\left(1 \times 10^{5}\right)$ were seeded in 6-well plates and treated with lupeol at 50 or $100 \mu \mathrm{M}$ for $72 \mathrm{~h}$. For cell cycle analysis, the cells were collected, washed with cold PBS, and fixed with cold $80 \%$ ethanol for $1 \mathrm{~h}$ at $4^{\circ} \mathrm{C}$. Subsequently, the cells were stained with $50 \mu \mathrm{g} / \mathrm{ml}$ of PI in the presence of $30 \mu \mathrm{g} / \mathrm{ml}$ DNase-free RNase A (Sigma-Aldrich) for $30 \mathrm{~min}$ at room temperature. The stained cell pellet was then resuspended in $500 \mu \mathrm{l}$ of PBS. The relative DNA content in each phase of the cell cycle was determined using a flow cytometer (FACSCalibeur, BD Biosciences) and CellQuest Pro software (version 5.1). For the Annexin V-PI double staining assay, the cells were harvested and double-stained with annexin V-FITC and PI using the Annexin V-FITC Apoptosis Detection kit I (BD Biosciences; PharMingen) according to the manufacturer's instructions. Annexin V-positive cells were determined using a flow cytometer and CellQuest software.

STAT3-luciferase reporter gene assay. The cells $\left(3 \times 10^{4}\right)$ were seeded in 24-well plates and co-transfected with $100 \mathrm{ng}$ of p-STAT3-TA-luc (Clontech) and $5 \mathrm{ng}$ of pRL-TK using Lipofectamine 2000 according to the manufacturer's instructions (Invitrogen; Thermo Fisher Scientific). At $24 \mathrm{~h}$ post-transfection, the cells were treated with lupeol for an additional 24 to $48 \mathrm{~h}$. The cells were then lysed and the STAT3 reporter gene activity was measured with the Dual-Luciferase Reporter Assay System (Promega) as described in the manufacturer's protocol.

EGFR stimulation by EGF treatment. The cells $\left(5 \times 10^{5}\right)$ were seeded in 6-well plates and treated with lupeol at 50 or $100 \mu \mathrm{M}$ for $24 \mathrm{~h}$. EGF (Lifeline Cell Technology) was then added at $20 \mathrm{ng} / \mathrm{ml}$ to the culture media $1 \mathrm{~h}$ prior o harvesting to activate EGFR.

Nuclear/cytosol extraction. To extract cytosolic fractions, $1 \times 10^{7}$ cells were lysed with buffer A [10 mM HEPES ( $\mathrm{pH} 7.9$ ), $1.5 \mathrm{mM} \mathrm{MgCl} 2,10 \mathrm{mM} \mathrm{KCl}, 0.5 \mathrm{mM}$ DTT, $1 \mathrm{mM}$ EDTA, $0.5 \%$ NP-40, protease inhibitor cocktail (Thermo Fisher Scientific), and phosphatase inhibitors $\left(1 \mathrm{mM} \mathrm{Na}_{3} \mathrm{VO}_{4}\right.$ and $100 \mathrm{mM} \mathrm{NaF}$ )] for $20 \mathrm{~min}$ on ice. The supernatant containing cytosolic proteins was collected by centrifugation $(900 \mathrm{x} \mathrm{g}$, $10 \mathrm{~min}, 4^{\circ} \mathrm{C}$ ) and cleared again by high-speed centrifugation $\left(16,000 \mathrm{x} \mathrm{g}, 10 \mathrm{~min}, 4^{\circ} \mathrm{C}\right)$. To extract the nuclear fraction, the pellet was washed with buffer A for three times and lysed with buffer $\mathrm{C}$ [20 mM HEPES ( $\mathrm{pH}$ 7.9), $1.5 \mathrm{mM} \mathrm{MgCl}{ }_{2}$, $420 \mathrm{mM} \mathrm{NaCl}, 0.2 \mathrm{mM}$ EDTA, $0.5 \mathrm{mM}$ DTT, 25\% glycerol, protease inhibitor cocktail (Thermo Fisher Scientific), and phosphatase inhibitors $\left(1 \mathrm{mM} \mathrm{Na}_{3} \mathrm{VO}_{4}\right.$ and $\left.\left.100 \mathrm{mM} \mathrm{NaF}\right)\right]$ for $1 \mathrm{~h}$ on ice with vigorous vortexing for $15 \mathrm{sec}$ every $10 \mathrm{~min}$. The supernatant containing nuclear proteins was obtained by centrifugation $\left(16,000 \mathrm{x} \mathrm{g}, 10 \mathrm{~min}, 4^{\circ} \mathrm{C}\right)$. To detect any cross-contamination between the nuclear and cytosolic fractions, Lamin B (1:1,000 dilution) and $\alpha$-tubulin (1:1,000 dilution) were used as markers for the nuclear and cytosolic fractions, respectively.

Constitutive activation of STAT3. The cells $\left(5 \times 10^{5}\right)$ were seeded in a 6-well plate and transfected with $1 \mu \mathrm{g}$ of pExpress1-stat3Y705D for the constitutive activation of STAT3, or with $1 \mu \mathrm{g}$ of pExpress-1 as a control, using Lipofectamine 2000. pExpress-1 and pExpress1-stat3Y705D were kindly provided by Professor Ho-Young Lee (Seoul National University). At $48 \mathrm{~h}$ post-transfection, the cells were trypsinized and seeded again in 6-well plates. The cells were treated with lupeol at $50 \mu \mathrm{M}$ for a further $72 \mathrm{~h}$, and subsequently examined by western blot analysis.

RT-PCR and semi-quantitative PCR. Total RNA was extracted using TRIzol reagent (Invitrogen; Thermo Fisher Scientific) according to the manufacturer's instructions. First-strand cDNA was synthesized with the PrimeScript RT reagent kit (Takara) using $1 \mu \mathrm{g}$ of total RNA as described in the manufacturer's protocol. The primer sequences used are as follows: Survivin forward, 5'-TCA AGG ACC ACC GCA TCT CTA-3' and reverse, 5'-TGA AGC AGA AGA AAC ACT GGG-3'; cyclin D1 forward, 5'-CCT GTC CTA CTA CCG CCT CA-3' and reverse, $5^{\prime}$-TCC TCC TCT TCC TCC TCC TC-3'; and actin forward, 5'-ACT ACC TCA TGA AGA TC-3' and reverse, 5'-GAT CCA CAT CTG CTG GAA-3'. cDNA was amplified using a SimpliAmp Thermal Cycler (Applied Biosystems). Cycle numbers corresponding to the exponential phase of the reaction were determined to be 28 cycles at an annealing temperature of $55^{\circ} \mathrm{C}$ for survivin and cyclin D1 and 20 cycles at an annealing temperature of $55^{\circ} \mathrm{C}$ for actin. The PCR products were resolved on a $1.5 \%$ agarose gel (Lonza), stained with nucleic acid gel staining solution (RBC), and visualized by the Gel Imaging System (Daihan Scientific).

Western blot analysis. The cells were lysed with cold radioimmunoprecipitation assay (RIPA) buffer (Thermo Fisher Scientific) supplemented with a protease inhibitor cocktail (Thermo Fisher Scientific) and phosphatase inhibitors (1 mM $\mathrm{Na}_{3} \mathrm{VO}_{4}$ and $100 \mathrm{mM} \mathrm{NaF}$ ) and incubated for $1 \mathrm{~h}$ on ice. The supernatants were collected by centrifugation at $16,000 \mathrm{x} \mathrm{g}$ at $4^{\circ} \mathrm{C}$ for $30 \mathrm{~min}$. Protein concentrations were determined using a bicinoconinic acid (BCA) protein assay kit (Pierce Biotechnology) according to the manufacturer's instructions. The same amounts $(20 \mu \mathrm{g})$ of protein were resolved by sodium dodecyl sulfate (SDS)-polyacrylamide gels (8-12\%) and transferred onto a polyvinyl difluoride (PVDF) membrane. The membrane was then blocked with $3 \%$ bovine serum albumin (BSA, GenDEPOT) in TBST [Tris-buffered saline (TBS) containing $0.1 \%$ Tween-20] for $1 \mathrm{~h}$ at room temperature and incubated overnight with primary antibodies (1:500 dilution for p-STAT3 antibody and p-EGFR antibody; 1:1,000 dilution for the other antibodies) at $4^{\circ} \mathrm{C}$. Following several washes with TBST for $1 \mathrm{~h}$, the membrane was incubated with secondary antibody solution (1:10,000 dilution in blocking solution) for $1 \mathrm{~h}$ at room temperature. Protein expression was detected by 
A
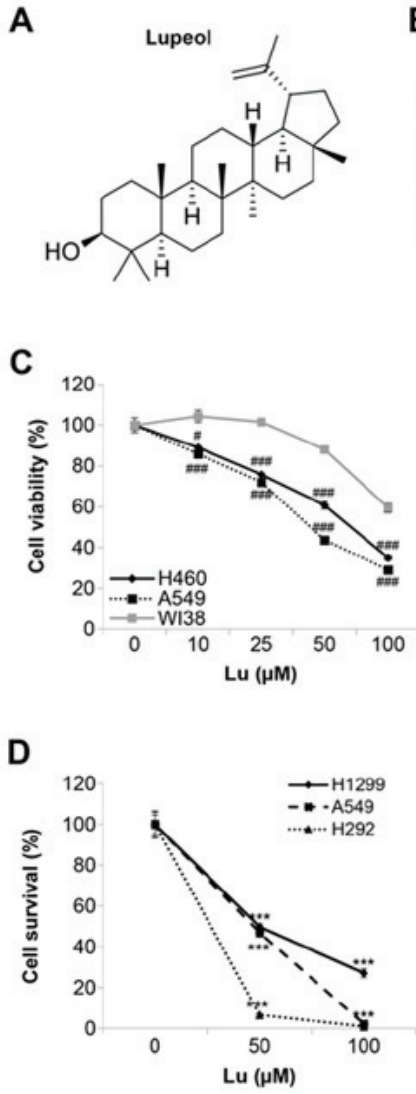

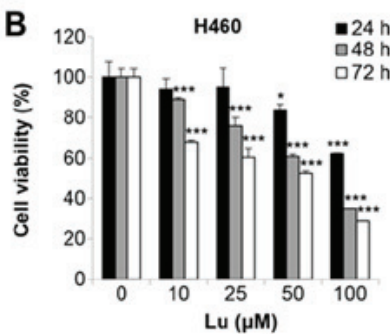

E

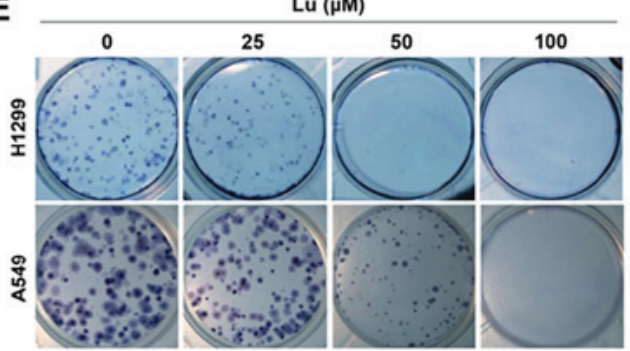

$\mathbf{F}$

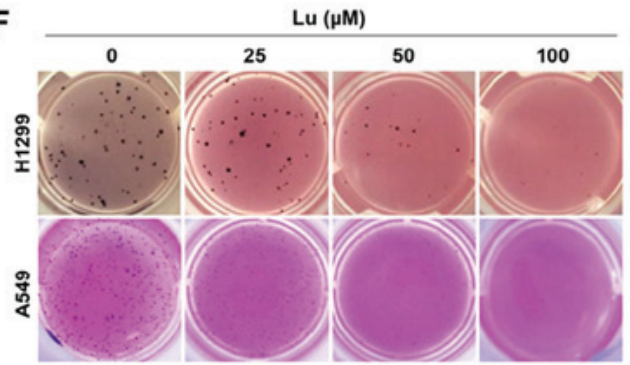

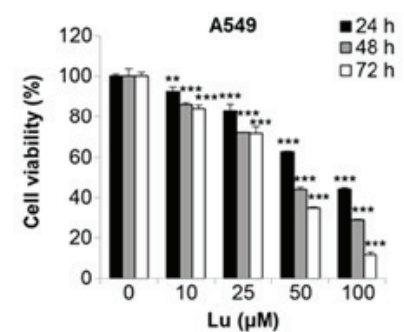
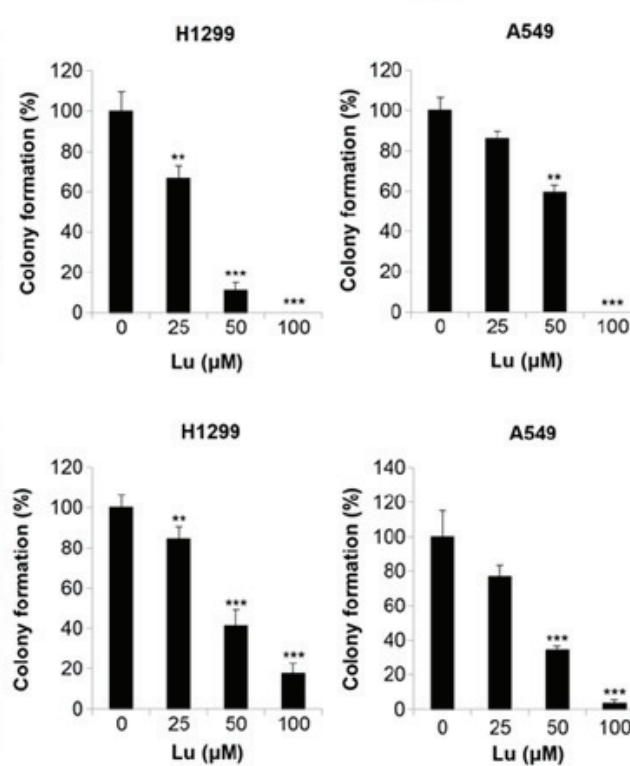

Figure 1. Effects of lupeol on the viability and colony formation of human NSCLC cells. (A) The structure of lupeol. (B) Various human NSCLC cell lines were treated with the indicated concentrations of lupeol for different incubation times. Cell viability was evaluated by MTT assay. (C) NSCLC cells and WI38 human lung fibroblasts were treated with lupeol for $48 \mathrm{~h}$. The cell viability was measured by the MTT assay. (D) NSCLC cells were treated with lupeol for $72 \mathrm{~h}$. Viable cells were counted by trypan blue exclusion assay. (E and F) Effects of lupeol on (E) anchorage-dependent and (F) anchorage-independent colony formation are shown. H1299 and A549 human NSCLC cells were seeded as a single-cell suspension in (E) 12-well plates or (F) in soft agar. Cells were grown for 2 weeks in medium containing lupeol. The colonies were visualized by a digital camera. The representative results of 3 independent experiments are shown (left panels). The number of colonies were counted using ImageJ software and normalized to untreated control cells (right panels). The data are expressed as the means \pm SD of 3 independent experiments. ${ }^{*} \mathrm{P}<0.05,{ }^{* *} \mathrm{P}<0.01$ and ${ }^{* * * *} \mathrm{P}<0.001$ vs. untreated controls; ${ }^{\#} \mathrm{P}<0.05$ and ${ }^{\# \# \#} \mathrm{P}<0.001$ vs. WI 38 cells treated with the indicated concentrations of lupeol. NSCLC, non-small cell lung cancer; Lu, lupeol.

SuperSignal West Pico Chemiluminescent Substrate (Thermo Fisher Scientific) according to the manufacturer's instructions. The densitometric analysis of the western blots was performed using Image J software (ImageJ 1.38; National Institutes of Health).

Molecular docking. The SwissDock web server (http://www. swissdock.ch) was used for molecular docking and prediction of the lowest free binding energy (38). The protein databank code (PDB) code for EGFR (1M17) was obtained from the Protein Data Bank (39). UCSF Chimera 1.13 software was used to explore the predicted binding modes. Among the clusters, the conformation with the lowest binding free energy was selected.

Statistical analyses. Each result is expressed as the mean \pm SD of data obtained from triplicate experiments. Statistical analyses were performed by Student's t-test or one-way ANOVA followed by a Tukey's post hoc test to determine the significant differences between groups. Differences with values of $\mathrm{P}<0.05$ were considered statistically significant. Statistical analysis was performed using GraphPad Prism 5 software (GraphPad Prism Software Inc.).

\section{Results}

Lupeol inhibits the growth and colony formation of human NSCLC cells. To examine the effects of lupeol on the growth of human NSCLC cell lines, the H1299, A549 and H460 cells were treated with various concentrations of lupeol for different periods of time. The results from MTT assay revealed that lupeol markedly reduced cell viability in a concentration- and time-dependent manner (Fig. 1B). We set $100 \mu \mathrm{g} / \mathrm{ml}$ as the maximum concentration of lupeol and $72 \mathrm{~h}$ as the optimal incubation time for further experiments. In order to examine the effects of lupeol on the viability of normal cells, we performed an MTT assay using the WI38 human lung fibroblasts. As shown in Fig. 1C, the viability of the WI38 fibroblasts was higher than that of the NSCLC cells, including the H460 and A549 cells, following $48 \mathrm{~h}$ of treatment with lupeol, indicating that lupeol exhibited a higher sensitivity to cancer cells than normal cells (Fig. 1C). To confirm the growth inhibitory effects of lupeol on NSCLC cells, trypan blue exclusion assays were performed on the H1299, A549 and H292 cells. The results revealed that the number of surviving cells was reduced by lupeol in a dose-dependent manner (Fig. 1D). These results clearly demonstrated that lupeol inhibited the growth of human NSCLC cells. 
A

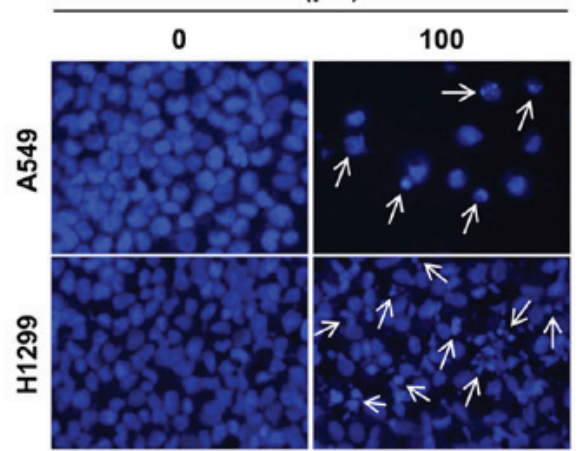

B
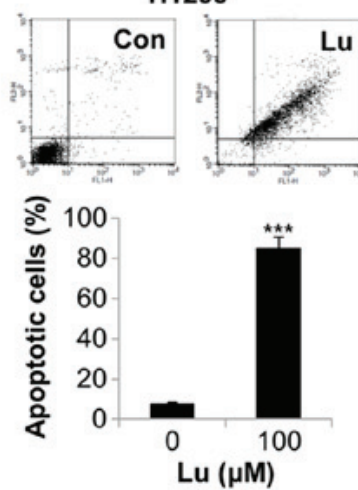

A549
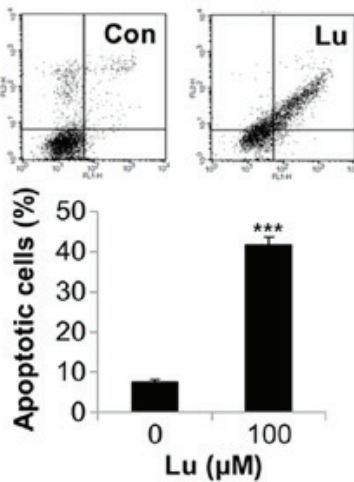

C
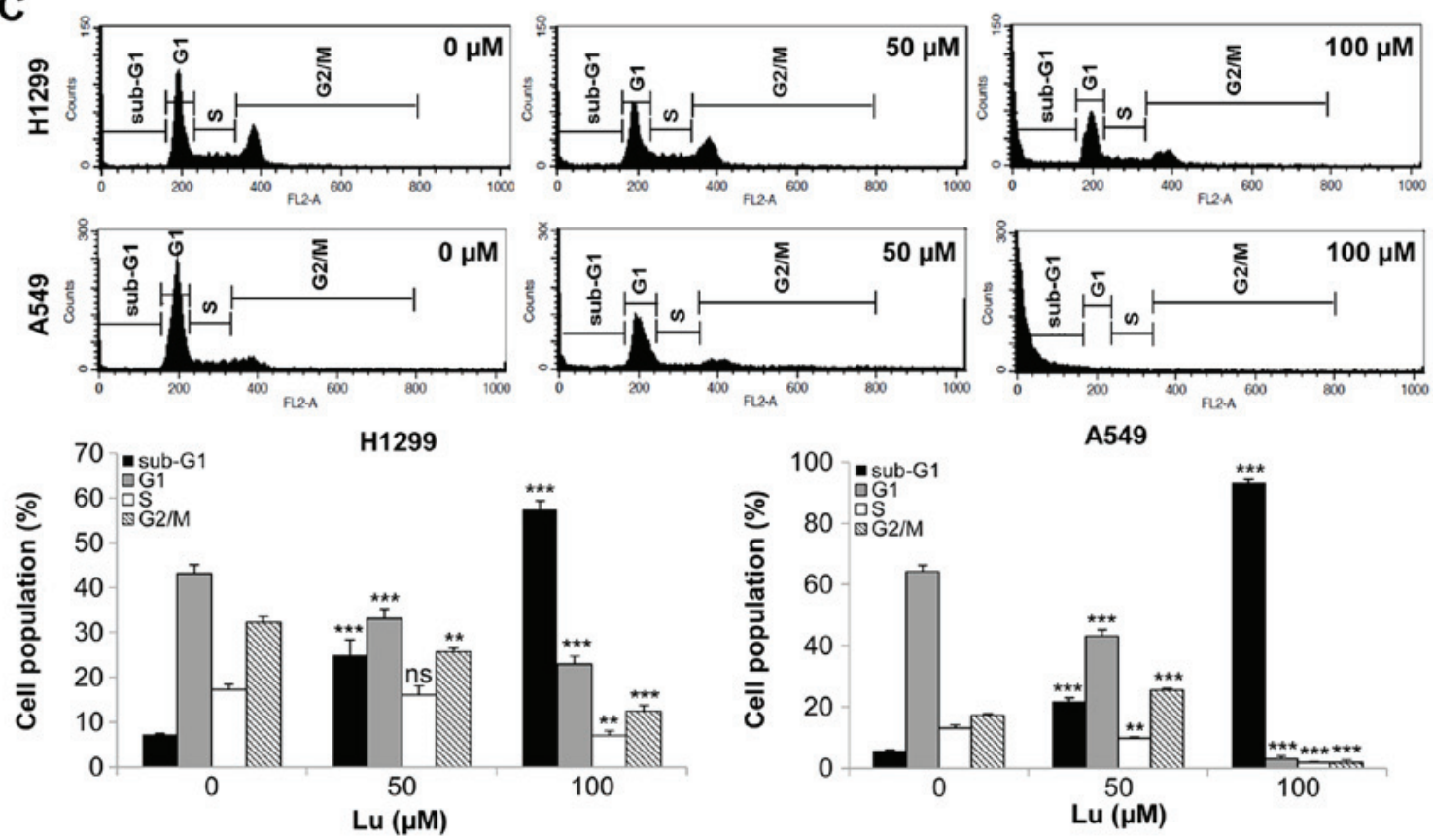

A549
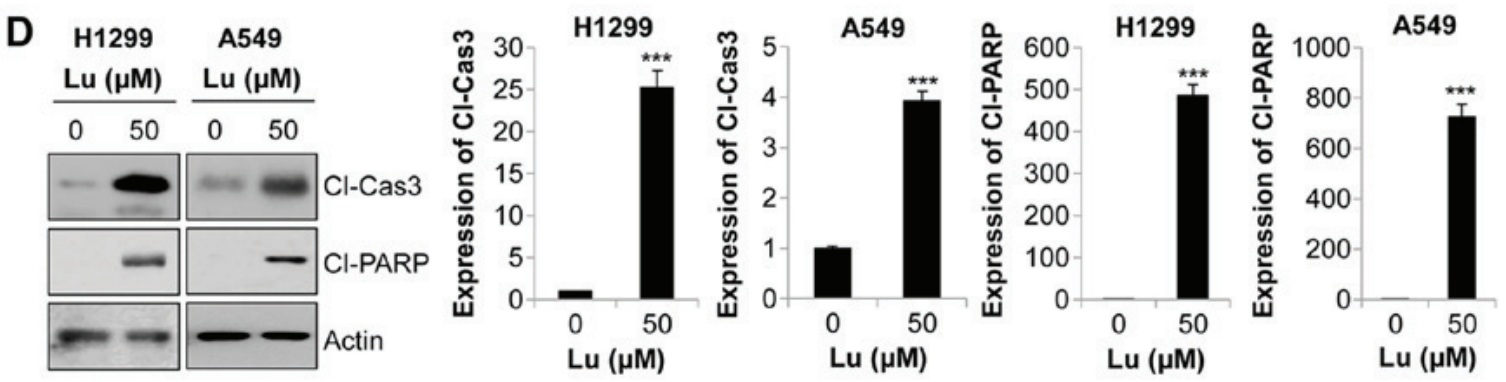

Figure 2. Induction of the apoptosis of human NSCLC cells by lupeol. H1299 and A549 cells were treated with lupeol for 72 h. (A) Nuclei were stained with DAPI solution. Stained nuclei were observed under a fluorescence microscope (x200 magnification). White arrows indicate the apoptotic cells. Representative fields of 3 independent experiments are shown. (B) The cells were double-stained with Annexin V-FITC and PI and analyzed using a flow cytometer. Annexin V-positive cells were identified as apoptotic cells. (C) The cells were stained with PI solution. The cell populations in sub-G1, G1, S, and G2/M phases were evaluated using a flow cytometer. The representative flow cytometry plots were shown. (D) The expression levels of cleaved PARP and cleaved caspase-3 were evaluated by western blot analysis. Actin was used as a loading control. The relative expression of cleaved caspase- 3 and cleaved PARP was analyzed with ImageJ software using actin for normalization. The data are expressed as the means \pm SD of 3 independent experiments. ns, not significant; ${ }^{* *} \mathrm{P}<0.01$ and ${ }^{* * *} \mathrm{P}<0.001$ vs. untreated controls. NSCLC, non-small cell lung cancer; Lu, lupeol; Cl-Cas3, cleaved caspase-3; Cl-PARP, cleaved PARP.

Subsequently, we examined the effects of lupeol on colony formation, a critical step in tumorigenesis, in human NSCLC cells. The results from the $2 \mathrm{D}$ colony formation assay indicated that lupeol significantly reduced the number of colonies in a dose-dependent manner in H1299 and A549 cells (Fig. 1E). To mimic the 3D tumorigenesis environment, a soft agar assay was further conducted. As shown in Fig. 1F, lupeol induced a dose-dependent decrease in colony formation in these cell lines (Fig. 1F). These results collectively indicated that lupeol suppressed anchorage-dependent and -independent colony formation in human NSCLC cells.

Lupeol induces the apoptosis of human NSCLC cells. To gain insight into the mechanisms underlying the anti-proliferative 
A

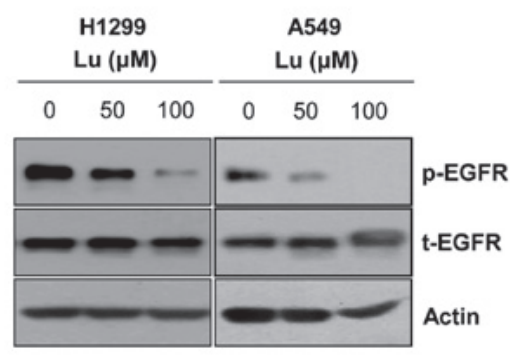

B

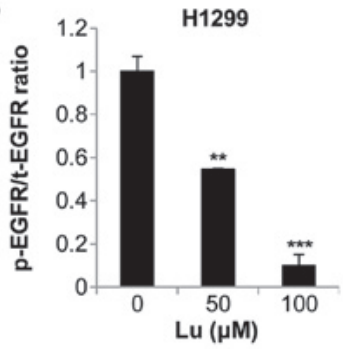

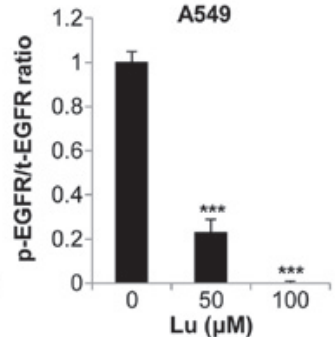

$\operatorname{Lu}(\mu \mathrm{M})$
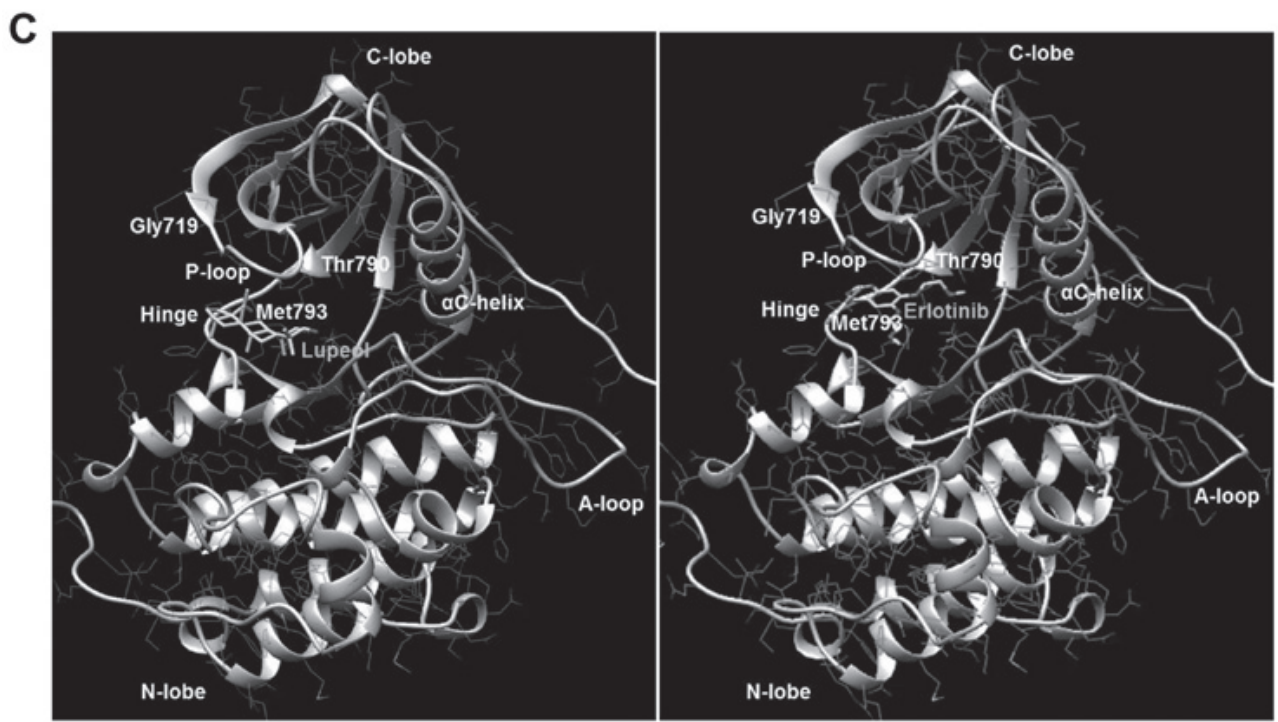

Figure 3. Effects of lupeol on EGFR activity in human NSCLC cells. (A and B) H1299 and A549 cells were treated with lupeol for $72 \mathrm{~h}$. (A) The expression levelss of p-EGFR and t-EGFR were evaluated by western blot analysis. Actin was used as a loading control. (B) The ratio of p-EGFR/t-EGFR was analyzed with ImageJ software using actin for normalization. The data are expressed as the means \pm SD of 3 independent experiments. ${ }^{* *} \mathrm{P}<0.01$ and ${ }^{* * *} \mathrm{P}<0.001 \mathrm{vs}$. untreated controls. (C) The interactions between EGFR and lupeol (left panel) or EGFR and erlotinib (right panel) were evaluated by molecular docking analysis using SwissDock. NSCLC, non-small cell lung cancer; EGFR, epidermal growth factor receptor; Lu, lupeol.

effects of lupeol on NSCLC cells, we performed DAPI staining. The NSCLC cells treated with lupeol exhibited highly condensed and fragmented nuclei, indicative of apoptotic cells (Fig. 2A). To confirm this result, we monitored apoptosis by flow cytometry. The results of Annexin V-PI double staining assay revealed that $72 \mathrm{~h}$ of treatment with lupeol markedly enhanced the Annexin V-positive cell population, an apoptotic portion, in NSCLC cells (Fig. 2B). Similar results were obtained by cell cycle analysis. The proportion of sub-G1 phase cells, i.e., apoptotic cells, was gradually increased in a dose-dependent manner (Fig. 2C). Likewise, the expression levels of cleaved PARP and cleaved caspase-3, apoptosis marker proteins, were upregulated by lupeol treatment (Fig. 2D). Taken together, these results demonstrated that lupeol triggered the apoptosis if human NSCLC cells.

Lupeol inhibits EGFR activation by direct binding to the EGFR TK domain in human NSCLC cells. As one of the pivotal oncogenic signaling pathways involved in NSCLC is EGFR, we then examined the effects of lupeol on the activity of EGFR. Western blot analysis indicated that the phosphorylation of EGFR was decreased by lupeol in a dose-dependent manner, while levels of the corresponding total proteins remained unaltered (Fig. 3A and B). To determine the mechanisms through which lupeol suppressed EGFR phosphorylation, we performed a molecular docking analysis using the SwissDock webservice (38). The crystal structure for the TK domain of EGFR [PDB ID: 1M17] was used for the analysis and erlotinib, a known EGFR TKI, was used as a control $(40,41)$. Five clusters with a total of 48 binding modes between lupeol and EGFR were generated and the binding $\Delta \mathrm{G}(-7.37$ to $-5.87 \mathrm{kcal} / \mathrm{mol})$ was calculated. Among the clusters, the conformation with the lowest binding $\Delta \mathrm{G}$ was selected. The $3 \mathrm{D}$ binding mode viewed by UCSF Chimera software clearly revealed that lupeol bound to the hinge region of the TK domain (Fig. 3C) with full fitness energy below $2,145 \mathrm{kcal} / \mathrm{mol}$, which was comparable to that of erlotinib (Table I). These results indicate that lupeol blocked EGFR activity by directly binding to the EGFR TK domain and competing with adenosine trisphosphate (ATP).

Inhibition of STAT3 activity by lupeol induces the apoptosis of human NSCLC cells. We further examined the effects of lupeol on the activity of downstream molecules of EGFR. As shown in Fig. S1, the phosphorylation levels of ERK and AKT were not decreased by lupeol treatment (Fig. S1). On the contrary, lupeol markedly suppressed the phosphorylation of STAT3 in NSCLC cells (Fig. 4A and B). To verify whether this event was dependent on EGFR regulation, we used EGF as a stimulator of EGFR. The phosphorylation of EGFR and STAT3 was enhanced by EGF treatment. However, the addition of lupeol reversed the EGF-mediated phosphorylation of EGFR and STAT3 (Fig. 4C). The same expression pattern of p-EGFR 
Table I. Docking of lupeol or erlotinib to the TK domain of EGFR (PDB ID: 1M17).

\begin{tabular}{llcc}
\hline Compound & \multicolumn{1}{c}{ Binding site } & Full fitness $(\mathrm{kcal} / \mathrm{mol})$ & Estimated $\Delta \mathrm{G}(\mathrm{kcal} / \mathrm{mol})$ \\
\hline Erlotinib & Hinge region of TK domain & $-2,200.16$ & -7.94 \\
Lupeol & Hinge region of TK domain & $-2,145.44$ & -7.37 \\
\hline
\end{tabular}
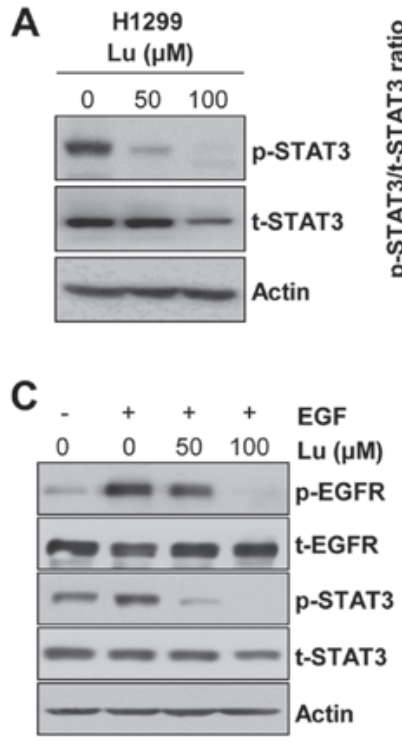

B

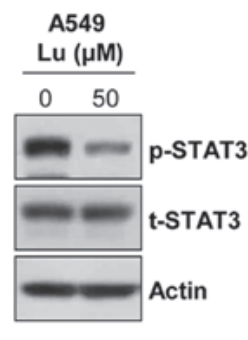

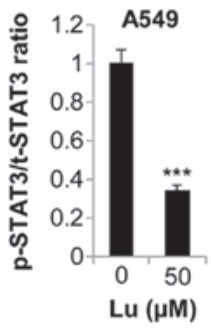

D

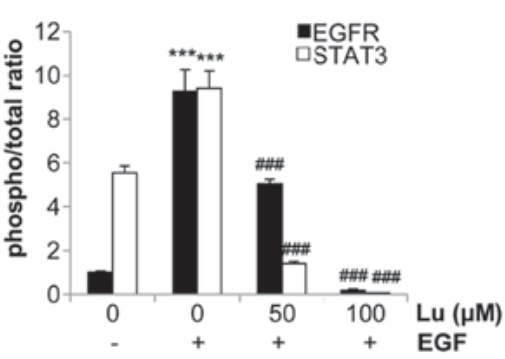

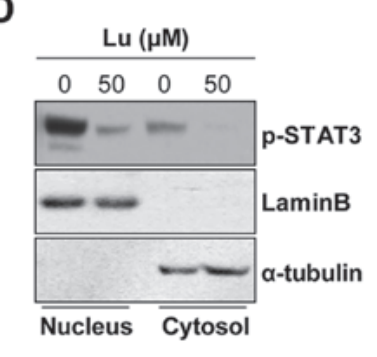
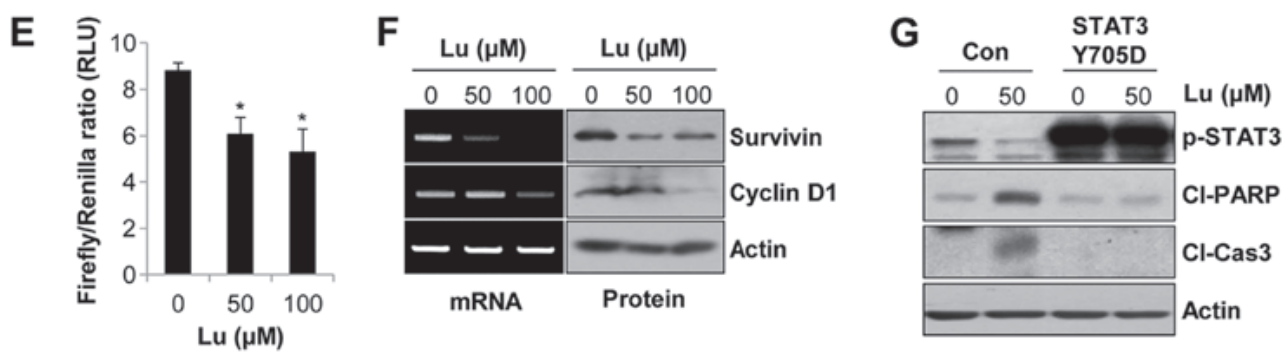

Figure 4. Suppression of the transcriptional activity of STAT3 by lupeol induces the apoptosis of human NSCLC cells. (A) H1299 and (B) A549 cells were treated with lupeol for $24 \mathrm{~h}$. The expression levels of p-STAT3 and t-STAT3 were detected by western blot analysis (left panels). The ratio of p-STAT3/t-STAT3 was calculated using Image J software (right panels). (C) A549 cells were treated with lupeol for $24 \mathrm{~h}$, and EGF (20 ng/ml) was then added to the culture media $1 \mathrm{~h}$ before harvesting to activate EGFR. The expression levels of the indicated proteins were evaluated by western blot analysis (left panel). The ratio of phosphorylated protein/total protein was analyzed with ImageJ software using actin for normalization (right panel). (D) H1299 cells were treated with lupeol for $24 \mathrm{~h}$. The nuclear translocation of p-STAT3 was examined by the nuclear/cytosol fractionation assay. Lamin B and $\alpha$-tubulin were used as markers of nuclear and cytosolic fractions, respectively. (E) H1299 cells were transfected with a STAT3-responsive Firefly luciferase construct (p-STAT3-TA-luc) and a Renilla luciferase construct (pRL-TK). At $24 \mathrm{~h}$ post-transfection, the cells were treated with lupeol for an additional $24 \mathrm{~h}$. The transcriptional activity of STAT3 was measured by the Dual-Luciferase Reporter Assay System. (F) H1299 cells were treated with lupeol for $72 \mathrm{~h}$. mRNA levels (left panel) and protein levels (right panel) of the STAT3 target genes were evaluated by RT-PCR (and semi-quantitative PCR) and western blot analysis, respectively. Actin was used as an internal control. (G) H1299 cells were transfected with control vector or STAT3 phospho-mimetic mutant (Y705D). At 48 h post-transfection, the cells were treated with the indicated concentrations of lupeol for an additional $72 \mathrm{~h}$. The expressions of p-STAT3, cleaved PARP and cleaved caspase-3 were detected by western blot analysis. Actin was used as an internal control. The data are expressed as the means \pm SD of 3 independent experiments. ${ }^{*} \mathrm{P}<0.05$ and ${ }^{* * *} \mathrm{P}<0.001$ vs. untreated controls; ${ }^{\# \# \# ~} \mathrm{P}<0.001$ vs. EGF-treated cells. NSCLC, non-small cell lung cancer; EGFR, epidermal growth factor receptor; Con, control vector; $\mathrm{Lu}$, lupeol; RLU, relative luciferase unit; $\mathrm{Cl}$-Cas3, cleaved caspase-3; Cl-PARP, cleaved PARP.

and p-STAT3 clearly indicated that lupeol suppressed STAT3 activity via EGFR regulation. In addition, the expression of p-STAT3 in the nuclear extracts was significantly decreased by lupeol in the H1299 cells, demonstrating that lupeol inhibited the nuclear translocation of p-STAT3 (Fig. 4D). The results from the dual luciferase reporter assay also revealed that the STAT3 reporter gene activity was reduced in a dose-dependent manner in the H1299 cells (Fig. 4E). Consistently, the mRNA and protein levels of STAT3 target genes, including survivin, an apoptosis-inhibitory protein, and cyclin D1, a protein involved in cell cycle progression, were decreased by lupeol in the H1299 cells (Fig. 4F). Taken together, these results indicated that lupeol blocked the transcriptional activity of STAT3 in human NSCLC cells.

We then examined whether the suppression of STAT3 activity by lupeol was sufficient to trigger the apoptosis of NSCLC cells. As shown in Fig. 4G, the H1299 cells transfected with pExpress1-stat3Y705D exhibited a significantly 

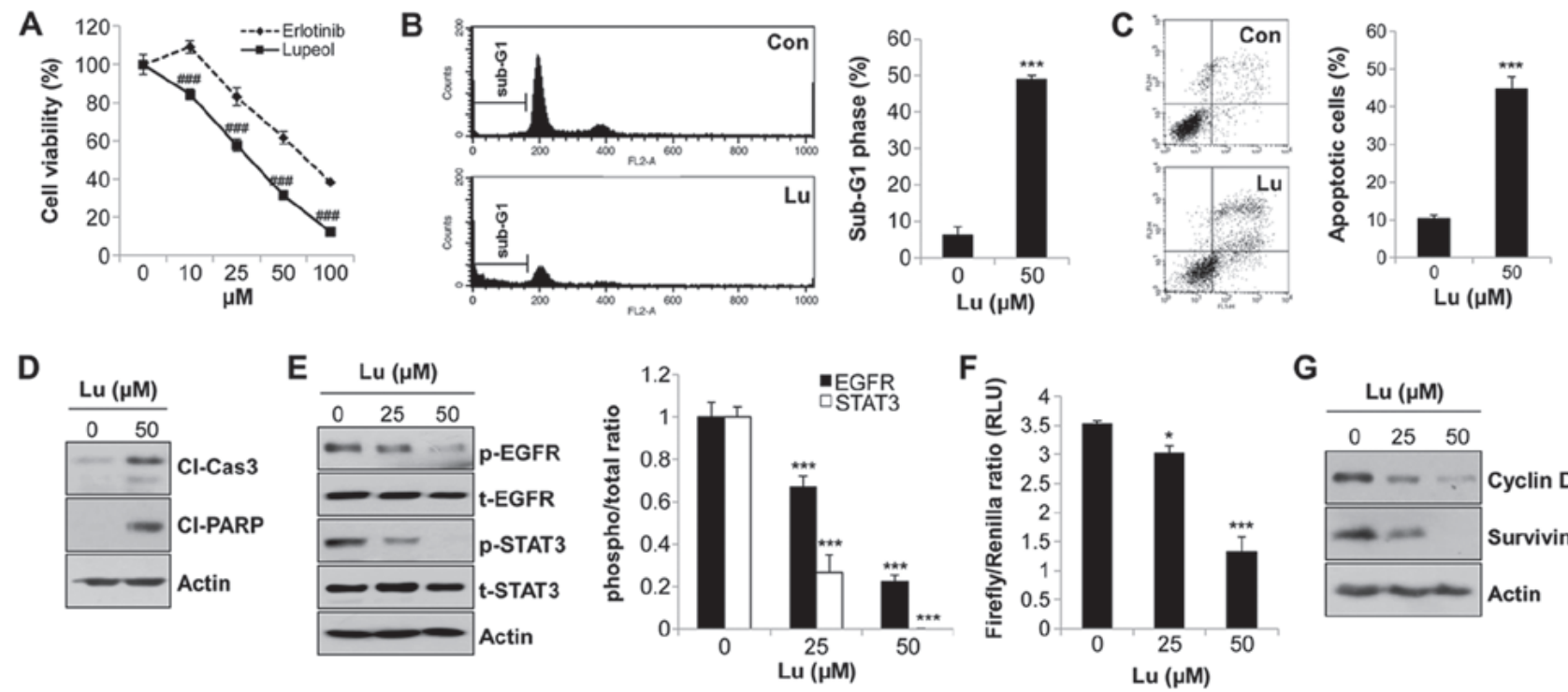

$G$

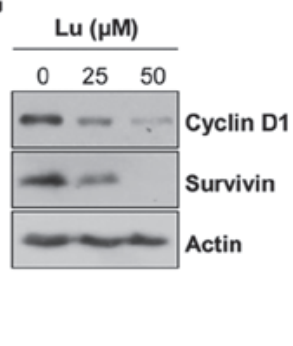

Figure 5. Anticancer effects of lupeol in erlotinib-resistant H1975 cells. (A) H1975 cells (EGFR L858R/T790M) were treated with lupeol or erlotinib for $72 \mathrm{~h}$. Cell viability was examined by MTT assay. (B-D) H1975 cells were treated with lupeol (50 $\mu \mathrm{M}$ ) for $72 \mathrm{~h}$. (B) The cells were stained with PI solution. Sub-G1 phase cells were evaluated by cell cycle analysis using flow cytometry. The representative flow cytometry plots were shown. (C) The cells were double-stained with annexin V-FITC and PI and analyzed using a flow cytometer. Annexin V-positive cells were identified as apoptotic cells. (D) The expression levels of cleaved PARP and cleaved caspase-3 were evaluated by Western blot analysis. (E) H1975 cells were treated with lupeol for $24 \mathrm{~h}$. The expression levels of the indicated proteins in the cell lysates were assessed by western blot analysis (left panel). The ratios of p-EGFR/t-EGFR and p-STAT3/t-STAT3 were analyzed with ImageJ software using actin for normalization (right panel). (F) H1975 cells were transfected with a STAT3-responsive Firefly luciferase construct and a Renilla luciferase construct. At $24 \mathrm{~h}$ post-transfection, the cells were treated with lupeol for an additional $48 \mathrm{~h}$. The transcriptional activity of STAT3 was measured by the Dual-Luciferase Reporter Assay System. (G) H1975 cells were treated with lupeol for $72 \mathrm{~h}$. The expressions of STAT3 target genes were evaluated by western blot analysis. Actin was used as a loading control. The data are expressed as the means \pm SD of 3 independent experiments. ${ }^{\# \# \#} \mathrm{P}<0.001$ vs. cells treated with erlotinib at indicated concentrations; ${ }^{*} \mathrm{P}<0.05$ and ${ }^{* * *} \mathrm{P}<0.001$ vs. untreated controls. NSCLC, non-small cell lung cancer; Con, control; Lu, lupeol; RLU, relative luciferase unit; Cl-Cas3, cleaved caspase-3; Cl-PARP, cleaved PARP.

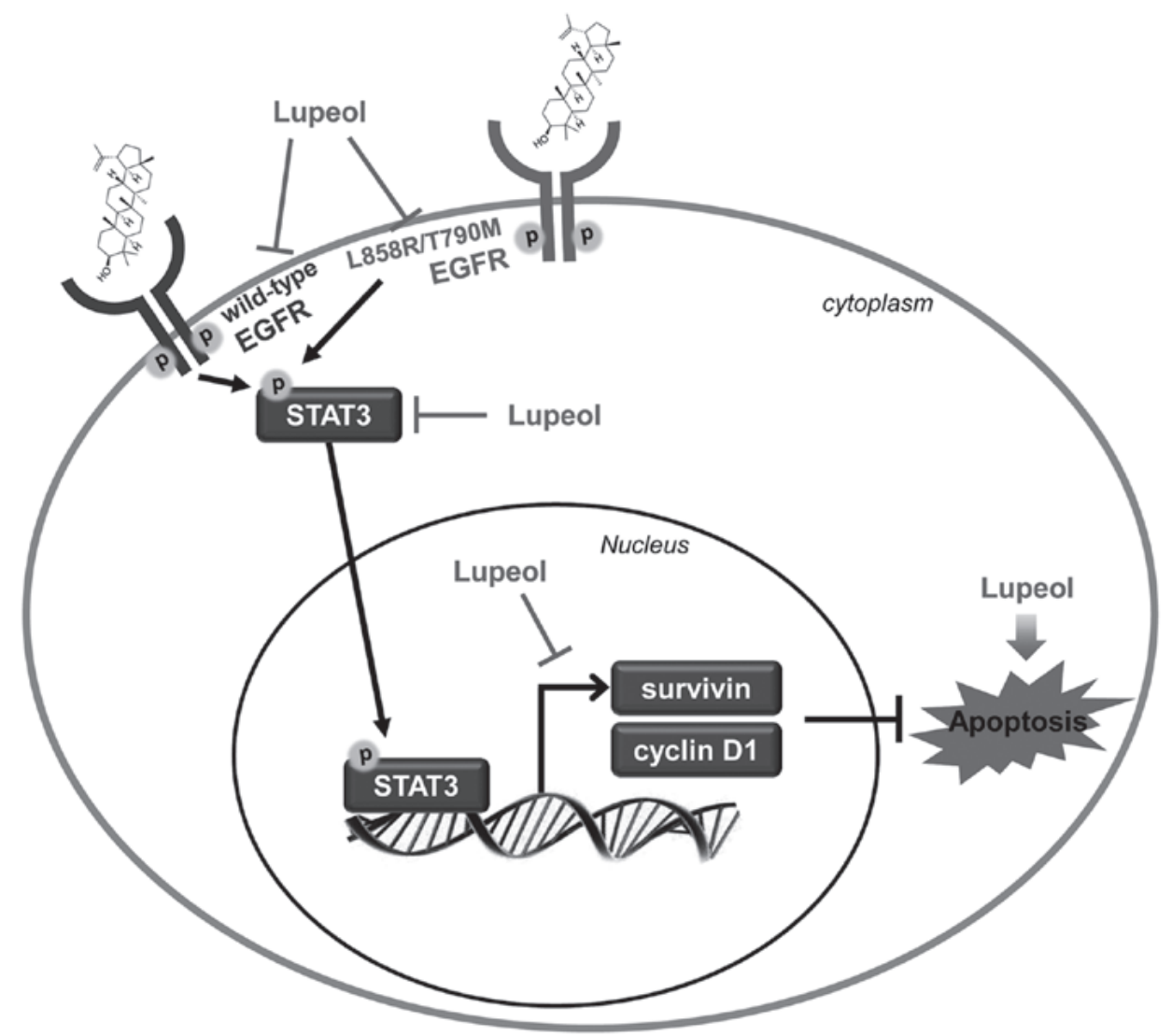

Figure 6. A schematic model of the molecular mechanism of lupeol-induced apoptosis in NSCLC cells. Lupeol exhibits anticancer activities, not only in EGFR wild-type NSCLC cells but also in erlotinib-resistant H1975 cells by the suppression of EGFR/STAT3 activity. NSCLC, non-small cell lung cancer; EGFR, epidermal growth factor receptor. 
upregulated p-STAT3 expression compared with that in cells transfected with the control vector. In addition, transfection with STAT3 Y705D downregulated the expression levels of cleaved PARP and cleaved caspase-3 which had been increased by lupeol (Fig. 4G). Thus, these data suggested that the inhibition of STAT3 activity by lupeol contributed to the induction of the apoptosis of NSCLC cells.

Lupeol exerts anticancer effects on EGFRTKI-resistant NSCLC cells. To examine whether lupeol exhibits anticancer activities in an EGFR TKI-resistant H1975 cell line with L858R/T790M double mutations, we first evaluated the effects of lupeol on the growth of H1975 cells. The results from MTT assay revealed that the viability of the erlotinib-resistant H1975 cells was markedly reduced by lupeol (Fig. 5A). Lupeol also increased the percentage of sub-G1 phase cells and Annexin V-positive cells (Fig. 5B and C) and upregulated the expression levels of cleaved PARP and cleaved caspase-3 in H1975 cells (Fig. 5D). These results suggested that lupeol suppressed the growth of H1975 cells by triggering apoptosis. We then verified whether these events were derived from the inhibition of the EGFR/STAT3 signaling pathway. Lupeol downregulated the expression levels of p-EGFR and p-STAT3 in a dose-dependent manner, while the corresponding total proteins remained unaltered in the H1975 cells (Fig. 5E). To measure the transcriptional activity of STAT3 following treatment with lupeol, the H1975 cells were transfected with a STAT3-responsive reporter vector and subsequently treated with lupeol for $48 \mathrm{~h}$. As shown in Fig. 5F, the STAT3 reporter gene activity was reduced in a dose-dependent manner (Fig. 5F). Consistently, the protein expression levels of STAT3 target genes, including cyclin D1 and survivin, were decreased by lupeol in the H1975 cells (Fig. 5G). Collectively, these results clearly demonstrated that lupeol exerted anticancer effects on EGFR TKI-resistant H1975 cells, as well as on EGFR wild-type NSCLC cells, through the suppression of EGFR/STAT3 activation (Fig. 6).

\section{Discussion}

The present study explored the potential anticancer effects of lupeol on NSCLC cells, focusing specifically on the regulation of the EGFR/STAT3 signaling pathway. Our results demonstrated that lupeol triggered the apoptosis of and inhibited the activity of EGFR and STAT3 in NSCLC cells, regardless of their EGFR mutation status. The novelty of this study is as follows: First, to the best of our knowledge, this is the first study to demonstrate a contribution of the EGFR/STAT3 axis to the lupeol-induced apoptosis of NSCLC cells. Although previous studies have reported that lupeol suppresses EGFR activity in several cancer cells, they usually identified AKT as a downstream target of EGFR $(36,37)$. In our case, the phosphorylation levels of AKT, as well as the MAPK proteins, the main signaling mediators activated by EGFR, were even slightly increased by lupeol treatment (Fig. S1). Instead, lupeol markedly suppressed the phosphorylation, nuclear translocation, and transcriptional activity of STAT3, suggesting that lupeol evoked anticancer effects in NSCLC cells by deactivation of the EGFR/STAT3 signaling pathway. Second, we reported the putative direct interaction between lupeol and EGFR. The docking analysis implemented by SwissDock revealed that lupeol effectively bound to the TK domain of EGFR with a low binding energy. Finally, we demonstrated that erlotinib-resistant H1975 cells were highly sensitive to lupeol. Lupeol consistently suppressed the activity of EGFR and STAT3 in H1975 cells. Given that the T790M mutation in EGFR accounts for approximately half of the acquired resistance to EGFR TKIs and lowers the response rate and overall survival of NSCLC patients $(16,17)$, our results suggest that lupeol may be a putative therapeutic option not only for EGFR TKI-naïve NSCLC patients, but also for advanced NSCLC with acquired resistance to EGFR TKIs.

Notably, STAT3 signaling has been implicated in primary and acquired resistance to EGFR TKIs. Various cell lines and ex vivo-based resistant models and patient-derived tumor tissue analyses have demonstrated that STAT3 activity may protect NSCLC cancer cells from EGFR TKI (22-27). These studies have suggested that STAT3 may be a desirable molecular target for enhancing sensitivity to EGFR TKIs. Our data also demonstrated that lupeol exerted potent anticancer effects in erlotinib-resistant H1975 cells via the suppression of STAT3 activation. The sole study that reported the inhibitory effects of lupeol on STAT3 activity was published by Siveen et al (7). They revealed that lupeol blocked the phosphorylation of STAT3 by enhancing the expression of tyrosine phosphatase SHP-2. In our case, lupeol did not affect the expression of SHP-2 and treatment with pervanadate, a phosphatase inhibitor, did not abrogate lupeol-induced apoptosis in NSCLC cells (data not shown). Instead, our results clearly demonstrated that STAT3 activation was dependent on the activity of EGFR.

Despite the novelty of this study, further molecular evidence should be provided to demonstrate the direct binding between lupeol and EGFR. We also cannot exclude the possibility that other upstream molecules of STAT3, such as IL6R and Src, are regulated by lupeol. In addition, if lupeol does modulate various upstream targets of STAT3, it would be of interest to examine the effects of lupeol on the crosstalk between these targets to strengthen its anticancer activity.

In conclusion, in this study, we propose a novel molecular mechanism of lupeol which involves the induction of the apoptosis of NSCLC cells. Our results revealed that lupeol can directly bind to the TK domain of EGFR with a quite low binding energy comparable to erlotinib, leading to the suppression of EGFR/STAT3 activity. Lupeol exerted anticancer effects, not only in EGFR wild-type NSCLC cells, but also on H1975 cells with a secondary T790M mutation. These results collectively suggest that lupeol may be an alternative therapeutic option for patients with advanced NSCLC with acquired EGFR-TKI resistance, as well as for patients with EGFR TKI-naïve NSCLC. Further preclinical and clinical studies are required to evaluate the anticancer activity of lupeol.

\section{Acknowledgements}

Not applicable.

\section{Funding}

This study was supported by grants from the National Research Foundation of Korea (NRF), Republic of Korea (No. NRF-2016R1C1B2015076). 


\section{Availability of data and materials}

All data generated or analyzed during this study are included in this published article. The datasets used and/or analyzed during the current study are available from the corresponding author on reasonable request.

\section{Authors' contributions}

SHP conceived and designed the study. TRM and HJP performed the majority of the in vitro experiments. SHP, KTH, GYC and YHC analyzed the data and coordinated the project. SHP, GYC and YHC wrote and reviewed the manuscript. All authors have read and approved the final manuscript.

\section{Ethics approval and consent to participate}

Not applicable.

\section{Patient consent for publication}

Not applicable.

\section{Competing interests}

The authors declare that they have no competing interests.

\section{References}

1. Keith RL and Miller YE: Lung cancer chemoprevention: Current status and future prospects. Nat Rev Clin Oncol 10: 334-343, 2013.

2. Blackhall FH, Shepherd FA and Albain KS: Improving survival and reducing toxicity with chemotherapy in advanced non-small cell lung cancer: A realistic goal? Treat Respir Med 4: 71-84, 2005.

3. Travis WD, Travis LB and Devesa SS: Lung cancer. Cancer 75 (Suppl): 191-202, 1995.

4. Mosesson Y and Yarden Y: Oncogenic growth factor receptors: Implications for signal transduction therapy. Semin Cancer Biol 14: 262-270, 2004.

5. Ohsaki Y, Tanno S, Fujita Y, Toyoshima E, Fujiuchi S, Nishigaki Y, Ishida S, Nagase A, Miyokawa N, Hirata S, et al: Epidermal growth factor receptor expression correlates with poor prognosis in non-small cell lung cancer patients with p53 overexpression. Oncol Rep 7: 603-607, 2000.

6. Inamura K, Ninomiya H, Ishikawa Y and Matsubara O: Is the epidermal growth factor receptor status in lung cancers reflected in clinicopathologic features? Arch Pathol Lab Med 134: 66-72, 2010.

7. Siveen KS, Nguyen AH, Lee JH, Li F, Singh SS, Kumar AP, Low G, Jha S, Tergaonkar V, Ahn KS, et al: Negative regulation of signal transducer and activator of transcription-3 signalling cascade by lupeol inhibits growth and induces apoptosis in hepatocellular carcinoma cells. Br J Cancer 111: 1327-1337, 2014.

8. Scagliotti GV, Selvaggi G, Novello S and Hirsch FR: The biology of epidermal growth factor receptor in lung cancer. Clin Cancer Res 10: S4227-S4232, 2004.

9. Veale D, Kerr N, Gibson GJ, Kelly PJ, Harris AL and Br J: The relationship of quantitative epidermal growth factor receptor expression in non-small cell lung cancer to long term survival. Br J Cancer 68: 162-165, 1993.

10. Fontanini G, De Laurentiis $M$, Vignati S, Chinè S, Lucchi M, Silvestri V, Mussi A, De Placido S, Tortora G, Bianco AR, et al: Evaluation of epidermal growth factor-related growth factors and receptors and of neoangiogenesis in completely resected stage I-IIIA non-small-cell lung cancer: Amphiregulin and microvessel count are independent prognostic indicators of survival. Clin Cancer Res 4: 241-249, 1998.
11. Ogawa J, Iwazaki M, Inoue $\mathrm{H}$, Koide $\mathrm{S}$ and Shohtsu A: Immunohistochemical study of glutathione-related enzymes and proliferative antigens in lung cancer. Relation to cisplatin sensitivity. Cancer 71: 2204-2209, 1993.

12. Shigematsu H and Gazdar AF: Somatic mutations of epidermal growth factor receptor signaling pathway in lung cancers. Int J Cancer 118: 257-262, 2006.

13. Lynch TJ, Bell DW, Sordella R, Gurubhagavatula S, Okimoto RA, Brannigan BW, Harris PL, Haserlat SM, Supko JG, Haluska FG, et al: Activating mutations in the epidermal growth factor receptor underlying responsiveness of non-small-cell lung cancer to gefitinib. N Engl J Med 350: 2129-2139, 2004.

14. Paez JG, Jänne PA, Lee JC, Tracy S, Greulich H, Gabriel S, Herman P, Kaye FJ, Lindeman N, Boggon TJ, et al: EGFR mutations in lung cancer: Correlation with clinical response to gefitinib therapy. Science 304: 1497-1500, 2004.

15. Pao W, Miller V, Zakowski M, Doherty J, Politi K, Sarkaria I, Singh B, Heelan R, Rusch V, Fulton L, et al: EGF receptor gene mutations are common in lung cancers from 'never smokers' and are associated with sensitivity of tumors to gefitinib and erlotinib. Proc Natl Acad Sci USA 101: 13306-13311, 2004.

16. Kobayashi S, Boggon TJ, Dayaram T, Jänne PA, Kocher O, Meyerson M, Johnson BE, Eck MJ, Tenen DG and Halmos B: EGFR mutation and resistance of non-small-cell lung cancer to gefitinib. N Engl J Med 352: 786-792, 2005.

17. Pao W, Miller VA, Politi KA, Riely GJ, Somwar R, Zakowski MF, Kris MG and Varmus H: Acquired resistance of lung adenocarcinomas to gefitinib or erlotinib is associated with a second mutation in the EGFR kinase domain. PLoS Med 2: e73, 2005.

18. Johnston PA and Grandis JR: STAT3 signaling: Anticancer strategies and challenges. Mol Interv 11: 18-26, 2011.

19. Harada D, Takigawa N and Kiura K: The role of STAT3 in non-small cell lung cancer. Cancers (Basel) 6: 708-722, 2014.

20. Jiang R, Jin Z, Liu Z, Sun L, Wang L and Li K: Correlation of activated STAT3 expression with clinicopathologic features in lung adenocarcinoma and squamous cell carcinoma. Mol Diagn Ther 15: 347-352, 2011.

21. Xu YH and Lu S: A meta-analysis of STAT3 and phospho-STAT3 expression and survival of patients with non-small-cell lung cancer. Eur J Surg Oncol 40: 311-317, 2014.

22. Gao SP, Chang Q, Mao N, Daly LA, Vogel R, Chan T, Liu SH, Bournazou E, Schori E, Zhang H, et al: JAK2 inhibition sensitizes resistant EGFR-mutant lung adenocarcinoma to tyrosine kinase inhibitors. Sci Signal 9: ra33, 2016.

23. Yao Z, Fenoglio S, Gao DC, Camiolo M, Stiles B, Lindsted T, Schlederer M, Johns C, Altorki N, Mittal V, et al: TGF-beta IL-6 axis mediates selective and adaptive mechanisms of resistance to molecular targeted therapy in lung cancer. Proc Natl Acad Sci USA 107: 15535-15540, 2010.

24. Li R, Hu Z, Sun SY, Chen ZG, Owonikoko TK, Sica GL, Ramalingam SS, Curran WJ, Khuri FR and Deng X: Niclosamide overcomes acquired resistance to erlotinib through suppression of STAT3 in non-small cell lung cancer. Mol Cancer Ther 12: 2200-2212, 2013

25. Jung MJ, Rho JK, Kim YM, Jung JE, Jin YB, Ko YG, Lee JS, Lee SJ, Lee JC and Park MJ: Upregulation of CXCR4 is functionally crucial for maintenance of stemness in drug-resistant non-small cell lung cancer cells. Oncogene 32: 209-221, 2013.

26. Fan W, Tang Z, Yin L, Morrison B, Hafez-Khayyata S, Fu P, Huang H, Bagai R, Jiang S, Kresak A, et al: MET-independent lung cancer cells evading EGFR kinase inhibitors are therapeutically susceptible to $\mathrm{BH} 3$ mimetic agents. Cancer Res 71: 4494-4505, 2011.

27. Zulkifli AA, Tan FH, Putoczki TL, Stylli SS and Luwor RB: STAT3 signaling mediates tumour resistance to EGFR targeted therapeutics. Mol Cell Endocrinol 451: 15-23, 2017.

28. Siddique HR and Saleem M: Beneficial health effects of lupeol triterpene: A review of preclinical studies. Life Sci 88: 285-293, 2011.

29. Wu XT, Liu JQ, Lu XT, Chen FX, Zhou ZH, Wang T, Zhu SP and Fei SJ: The enhanced effect of lupeol on the destruction of gastric cancer cells by NK cells. Int Immunopharmacol 16: 332-340, 2013.

30. Liu Y, Bi T, Dai W, Wang G, Qian L, Shen G and Gao Q: Lupeol enhances inhibitory effect of 5-fluorouracil on human gastric carcinoma cells. Naunyn Schmiedebergs Arch Pharmacol 389: 477-484, 2016.

31. Tarapore RS, Siddiqui IA, Adhami VM, Spiegelman VS and Mukhtar H: The dietary terpene lupeol targets colorectal cancer cells with constitutively active Wnt/ $\beta$-catenin signaling. Mol Nutr Food Res 57: 1950-1958, 2013. 
32. Pitchai D, Roy A and Ignatius C: In vitro evaluation of anticancer potentials of lupeol isolated from Elephantopus scaber L. on MCF-7 cell line. J Adv Pharm Technol Res 5: 179-184, 2014.

33. Sankaranarayanan S, Bama P, Sathyabama S and Bhuvaneswari N: Anticancer compound isolated from the leaves of Tridax procumbens against human lung cancer cell A-549. Asian J Pharm Clin Res 6: 91-96, 2013.

34. He W, Li X and Xia S: Lupeol triterpene exhibits potent antitumor effects in A427 human lung carcinoma cells via mitochondrial mediated apoptosis, ROS generation, loss of mitochondrial membrane potential and downregulation of m-TOR/PI3Ksol;AKT signalling pathway. J BUON 23: 635-640, 2018.

35. Lee TK, Castilho A, Cheung VC, Tang KH, Ma S and Ng IO: Lupeol targets liver tumor-initiating cells through phosphatase and tensin homolog modulation. Hepatology 53: 160-170, 2011

36. Liu Y, Bi T, Shen G, Li Z, Wu G, Wang Z, Qian L and Gao Q: Lupeol induces apoptosis and inhibits invasion in gallbladder carcinoma GBC-SD cells by suppression of EGFR/MMP-9 signaling pathway. Cytotechnology 68: 123-133, 2016.
37. Rauth S, Ray S, Bhattacharyya S, Mehrotra DG, Alam N, Mondal G, Nath P, Roy A, Biswas J and Murmu N: Lupeol evokes anticancer effects in oral squamous cell carcinoma by inhibiting oncogenic EGFR pathway. Mol Cell Biochem 417: 97-110, 2016

38. Grosdidier A,Zoete V and Michielin O: SwissDock, a protein-small molecule docking web service based on EADock DSS. Nucleic Acids Res 39 (Suppl): W270-7, 2011.

39. Berman HM, Westbrook J, Feng Z, Gilliland G, Bhat TN, Weissig H, Shindyalov IN and Bourne PE: The protein data bank. Nucleic Acids Res 28: 235-242, 2000.

40. Choowongkomon K, Sawatdichaikul O, Songtawee N and Limtrakul J: Receptor-based virtual screening of EGFR kinase inhibitors from the NCI diversity database. Molecules 15: 4041-4054, 2010.

41. Stamos J, Sliwkowski MX and Eigenbrot C: Structure of the epidermal growth factor receptor kinase domain alone and in complex with a 4-anilinoquinazoline inhibitor. J Biol Chem 277: 46265-46272, 2002. 